\title{
Mixed-list manipulations of implicit associative responses in verbal discrimination learning: II. Strategies and interactions with rate of presentation
}

\author{
N. JACK KANAK, BIJAN RABENOU, and JAY L. OLSON \\ University of Oklahoma, Norman, Oklahoma 73069
}

\begin{abstract}
Implicit associative responses were manipulated in a verbal discrimination task involving a mixed list of intrapair, interpair, interitem-AR, and control conditions in combination with three rates of presentation $(1: 1,2: 2$, and $4: 4 \mathrm{sec})$. The intrapair condition was significantly superior to the control at all three presentation rates. The interpair condition was inferior to the control at the $1: 1$ sec rate, equal to the control at the $2: 2$ rate, and superior at the $4: 4$ rate. The interitem-AR condition was equal to the control at the $1: 1 \mathrm{sec}$ rate, but superior at the $2: 2$ and $4: 4$ rates. The results were interpreted as consistent with the hypothesis that multiple higher order strategies may be invoked in such a mixed list design and that these strategies differ in levels of complexity, resulting in a complex interaction with rate of presentation.
\end{abstract}

According to the frequency theory of verbal discrimination (VD) learning (Ekstrand, Wallace, \& Underwood, 1966), the presence of associatively related items has a facilitative or an inhibitory effect on the acquisition of a VD list depending on whether the implicit associative response (IAR; Bousfield, Whitmarsh, \& Danick, 1958) acts to increase or to decrease the intrapair subjective frequency differential between the right (R) and the wrong (W) items of a pair. The theory predicts that the learning of lists containing associatively related $\mathbf{W}$ and $\mathrm{R}$ items in two different pairs (an interpair condition), or within a single pair (an intrapair condition) would be more difficult than learning a control list of associatively unrelated items, since the differential frequency cue is assumed to be reduced by the increment in subjective frequency of $\mathrm{W}$ items, in the interpair and intrapair conditions, caused by the IAR to the $\mathrm{R}$ items. When the list contains associatively related $\mathbf{R}$ items and unrelated $\mathbf{W}$ items (an interitem- $A R$ condition), however, learning should be facilitated relative to the control condition.

Eckert and Kanak (1974) and Kanak and Jones (1974) have presented reviews of the literature regarding these three classes of IAR manipulations. Substantial support for the prediction of interference for the interpair condition has been obtained in several studies. The intrapair condition, however, has produced conflicting results with some investigators (e.g., McCarthy, 1973; Palermo \& Ullrich, 1968) supporting frequency theory while several other experiments have shown null differences when compared to the control condition. Among the latter, Fulkerson and Kausler

This research was supported in part by funds provided by the University Research Council of the University of Oklahoma. Requests for reprints should be addressed to N. Jack Kanak, Department of Psy chology, University of Oklahoma, Norman, Oklahoma 73069. The authors thank Marla Jardon for typing the manuscript.
(1969) suggested that subjects employ a "tagging" mechanism, compensatory to the reduction of the differential frequency cue which results from the IAR. According to the tagging hypothesis, after the subject recognizes the presence of intrapair associations, during the feedback interval he uses the strategy of "tagging" the underlined $\mathrm{R}$ item as "correct" and then collapses the tag over the association for memory storage (e.g., rehearses "table-chair," "chair is correct"). On the subsequent anticipation or test trial, the subject needs only to recognize the rehearsed intrapair association ("table-chair") upon presentation of the pair and then retrieve from memory the "tag" ("chair is correct"). Kanak and Jones (1974) noted that two potentially important methodological differences exist between those studies of the intrapair condition which support frequency theory and those which do not. Those supporting the theory used 1:1 presentation rates and a mixed list of intrapair and control pairs while those not supporting the theory used $2: 2-\mathrm{sec}$ rates and unmixed lists. Either or both of these variables could be responsible for the discrepant results since (a), as Lovelace and Schultz (1971) have pointed out, a mixed-list manipulation may produce selective and differential attention to the attributes of the materials, and (b) a 1:1-sec rate may not allow adequate time for the utilization of a compensatory tagging process as compared to a 2:2-sec rate. Employing a 2:2-sec rate of presentation and a deliberate strategy of using a mixed list of not only intrapair and control pairs, but also interpair and interitem-AR pairs, to induce selective attention, Kanak and Jones (1974) found the intrapair condition significantly superior to the control condition as predicted by the tagging hypothesis.

With regard to the interitem-AR condition, Ekstrand et al. (1966) reported significant facilitation relative to the control condition as predicted by frequency theory. 
However, in only one of seven other experiments (cf. Kanak \& Jones, 1974) reported in the literature has this result been replicated, and only then when the subjects were instructed regarding the associative relationship (Kanak, Cole, \& Eckert, 1972; Experiment II).

The operation of the IAR mechanism per se in interpair and intrapair manipulations, though possibly in a different manner than that predicted by frequency theory in some studies dealing with the latter condition, seems well established. Thus the lack of consistent evidence for its operation in the in teritem-AR condition is paradoxical. Kanak et al. (1972) pointed out that the interpair and intrapair manipulations both involve a contrast of item function (i.e., $W$ or $R$ ) of the associatively related items which may activate awareness of and attention to the presence of associations even in unmixed lists. In an unmixed list interitem-AR condition, however, as Kanak and Jones have noted, such an item-function contrast cue is not present to provide the same cue for the subjects to attend to the associative relationship. These authors hypothesized that their mixed list procedure involving intrapair and interpair items, with their possible item-function contrast cues, would produce a generalization of attention to associative relationships in the interitem-AR condition. Consistent with this hypothesis, the interitem-AR condition was found to be significantly superior to the control condition as frequency theory predicts.

Kanak and Jones failed to find the interpair condition inferior to the control, contrary to previous studies using unmixed lists. They noted, however, that the subject could possibly utilize his awareness of the positive contribution of associates in the intrapair and interitem-AR conditions to minimize the inhibitory influence, once experienced, of the associates in the interpair condition. These authors further suggested that, in a mixed list involving several treatment manipulations, if the subject is capable of utilizing a process of a higher level than simple differential frequency unit accrual as the results of the intrapair condition suggested, he may attempt to apply higher order strategies to all types of pairs involving associates. Such multiple strategies might involve the subject's attending to the associates if they involve $\mathrm{W}$ and $\mathrm{R}$ items in the same pair, using a "tagging" strategy; attending to them if the associates involve only $\mathbf{R}$ items, using an "approach" strategy (Cole \& Kanak, 1972); and ignoring associations and relying on rote memory if they involve $\mathrm{W}$ and $\mathrm{R}$ items in different pairs, a strategy Cole and Kanak suggested was involved in their "instructed" interitem-AW condition. In the latter case, the interpair condition would not be expected to differ from the control. A second possible strategy in the interpair condition is to utilize a "tagging"-like approach with the subject labeling "chair is correct," "table is incorrect" and recalling the appropriate tag to mediate his response when either of the pairs is presented involving a given interpair association.

The present experiment represents an extension of the Kanak and Jones study designed to test whether such multiple strategies may be employed by varying rate of presentation. In terms of the complexity of the higher order processes suggested for the various conditions, that for the interpair condition seems intuitively most complex and should therefore be most sensitive to time factors. A rapid rate of presentation (e.g., 1:1 sec) may not allow adequate time for the employment of higher order strategies and, if so, frequency based predictions should be supported, particularly for the intrapair and interpair conditions which involve the item function contrast. With slower rates of presentation (e.g., 2:2 and $4: 4 \mathrm{sec}$ ), increasing support for the use of higher order strategies in the form of both improvement across rates of presentation and improved performance within each IAR condition, relative to the control, is predicted.

\section{METHOD}

\section{Design and Subjects}

The experimental design was a 3 by 4 factorial with three rates of presentation $(1: 1,2: 2$, and $4: 4 \mathrm{sec})$ as a between-subject variable and four types of pairs (intrapair, interpair, interitem-AR, and control) as a within-subject variable. Seventy-two students from introductory psychology classes at the University of Oklahoma participated in the experiment as an option for partial fulfillment of a course requirement. All subjects were naive to VD learning. The subjects were randomly assigned to the three rate of exposure conditions $(n=24)$ and to the various forms of the lists within each condition $(n=2)$ upon their order of appearance at the laboratory in a random block assignment procedure.

\section{Lists and Procedure}

The word lists and their various counterbalanced forms were identical to those described by Kanak and Jones (1974) involving 20 pairs with four each of the intrapair, interpair, and control types and eight in the interitem-AR condition. Eight interitem-AR pairs were employed in order to represent four associative relationships, as in the other two experimental conditions. The intertrial interval was $4 \mathrm{sec}$ for all conditions. All other aspects of the procedure were identical to the Kanak and Jones study except that the criterion for learning was one perfect trial rather than two.

\section{RESULTS}

Consistent with Kanak and Jones, the data for total errors were reduced to the proportion of errors to the base of opportunities to provide a common index, because of the differential number of pairs to be learned among the treatment conditions. The total number of errors to the criterion of one perfect trial was tabulated for each subject for those pairs within each treatment condition, and this value was divided by the product of the number of items in that condition multiplied by the number of trials the subject required to achieve the criterion for all pairs in that condition.

Table 1 presents the mean proportion of errors to the 
Table 1

Mean Proportion of Errors to the Base of Opportunities for Each Type of Pair and Rate of Presentation

\begin{tabular}{ccccc}
\hline $\begin{array}{c}\text { Rate of } \\
\text { Presen- } \\
\text { tation } \\
\text { (Seo) }\end{array}$ & Control & $\begin{array}{c}\text { Intra- } \\
\text { pair }\end{array}$ & $\begin{array}{c}\text { Inter- } \\
\text { pair }\end{array}$ & $\begin{array}{c}\text { Tnter- } \\
\text { item } \\
\text { AR }\end{array}$ \\
\hline $1: 1$ & .267 & .181 & .321 & .250 \\
$2: 2$ & .262 & .181 & .263 & .203 \\
$4: 4$ & .271 & .175 & .216 & .223 \\
\hline
\end{tabular}

base of opportunities for each of the 12 treatment conditions. A mixed 3 by 4 analysis of variance produced a highly significant effect for types of pairs $[F(3 / 207)=17.28, \quad p<.001]$ and a significant interaction of type of pair with rate of presentation $[F(6 / 207)=2.38, p<.05]$. The main effect of rate of presentation did not reach significance $(\mathrm{p}>.10)$.

Multiple comparisons on the interaction means across types of pairs within each rate of presentation were conducted by the Newman-Keuls test. At the 1:1-sec rate the intrapair condition produced a significantly lower error rate than all the other three types of pairs (all ps $<.01$ ), while the interpair condition had a higher error rate than all other three groups (all ps $<.05$ ). No other differences were significant. Performance on the intrapair condition was significantly higher than the interpair and control conditions (both ps $<.01$ ) at the $2: 2$-sec rate, but the intrapair and interitem-AR conditions did not differ significantly. In addition, the interpair condition resulted in a higher error rate than the intrapair and interitem-AR conditions (both ps $<.05$ ), but no significant difference was obtained between the interpair and control pairs. Furthermore, the interitem-AR condition was significantly superior to the control condition $(p<.05)$. These results at the $2: 2$-sec rate are consistent with the conclusions of Kanak and Jones (1974) and hence constitute a replication of those results. At the 4:4-sec rate, the intrapair, interpair, and interitem-AR conditions were all superior to the control condition ( $p s<.05)$, but did not differ from each other.

The main effect of rate of presentation was not significant, as noted above. In fact, Table 1 indicates the mean error rates for the control conditions, as well as the intrapair conditions, across the three rates of presentation were almost identical. This lack of an effect for rate of presentation in the control condition is in apparent contradiction with previous findings that slower rates produce superior performance in lists of unrelated items (e.g., Kanak, 1968). It should be noted, however, that total errors to the base of opportunities is a relatively insensitive measure of rate effects, especially when the number of trials to reach criterion is small. This is particularly the case when the first trial is a guessing trial with a $50 \%$ chance of error, as was the case in the present experiment. To confirm that rate of presentation had a significant effect of the speed of learning control pairs, a simple analysis of variance of the number of trials to criterion for control pairs was conducted. This analysis resulted in a highly significant effect $[F(2 / 69)=22.45, p<.0001]$. The means were $20.50,8.50$, and 7.13 for the $1: 1,2: 2$, and $4: 4$-sec rates, respectively. The $1: 1-\mathrm{sec}$ rate differed significantly from the other two rates (both ps $<.01$ ) which did not differ from each other.

\section{DISCUSSION}

The intrapair condition proved significantly superior to the control condition at all three rates of presentation. This finding is in opposition to frequency theory and to the results of Palermo and Ullrich (1968) and McCarthy (1973), who used the $1: 1-\mathrm{sec}$ presentation rate but also used a mixed list. It is well to note, however, that the mixed list used by these investigators included only two types of intrapair pairs and one type of control pair, while the present study also employed interpair and interitem-AR treatments. If mixed lists do induce selective attention to the materials (Lovelace \& Schultz, 1971), then the addition of two different types of treatment pairs may further increase the possibility of selective attention. If so, the attention of the subjects may have been focused on the associative relationship among the items of the intrapair condition at an earlier stage of learning, thus allowing a "tagging" strategy to be invoked even at the $1: 1-\mathrm{sec}$ rate. The efficiency of employment of such a strategy did not, however, improve across rates of presentation. This suggests that if "tagging" was indeed the strategy, it is a relatively simple strategy to use in terms of its level of complexity.

Consistent with the frequency theory, and previous studies employing unmixed lists (e.g., Eberlein \& Raskin, 1968; Fulkerson \& Kausler, 1969; Lovelace \& Schulz, 1971) and $2: 2-\mathrm{sec}$ presentation rates, the interpair condition was significantly inferior to the control condition at the $1: 1$-sec rate. The nonsignificant difference between these two conditions at the 2:2-sec rate replicates the results of Kanak and Jones and suggests that some mechanism was operative that was at least partially compensatory to the effects predicted by frequency theory, when compared to the results of the $1: 1-\mathrm{sec}$ rate. This suggestion receives further support in the superiority of the interpair condition at the $4: 4$-sec rate, a result providing some credence for the hypothesized, more complex form of "tagging" possible in this condition.

The interitem-AR condition produced a null difference at the $1: 1-\mathrm{sec}$ rate. While this result does not support frequency theory or an "approach" strategy, the interpair condition did support frequency theory at this rate while the intrapair condition yielded results consistent with a tagging notion. The interitem-AR condition, however, was superior to the control at the 2:2- and 4:4-sec rates. While this result can be taken as support for either frequency theory or an approach strategy, it may be noted that at the $2: 2-\mathrm{sec}$ rate the interpair condition had improved to the level of not differing from the control, and the intrapair condition maintained its superiority. At the 4:4-sec rate, the interaction effect breaks down completely and all three IAR conditions become significantly superior to the control condition.

Viewing this analysis of the results as a pattern presents substantial support for the initial hypothesis that multiple higher order strategies are possible in a mixed list employing these treatment conditions. However, the data also support the view that these strategies differ in complexity, and hence their efficiency of utilization interacts with exposure time. The simple "tagging" strategy suggested as involved in the intrapair 
condition is viewed as the least complex, and hence employed even with minimal time, while the interpair strategy of tagging one associate as correct in one pair and the other as incorrect in another pair is the most complex and requires more processing time. The lesser efficiency of an "approach" strategy, if such is involved in the interitem-AR condition. relative to the tagging strategy of the intrapair condition, may be due to the lack of an item-function contrast cue in the interitem-AR condition, as noted earlier, which makes subjects of this condition dependent on the generalization of the awareness of associated items in the intrapair and interpair conditions to the interitem-AR condition before an "approach strategy" emerges.

Finally, although the particular pattern of interactions of types of pairs and rate of presentation may or may not be obtained when these variables are manipulated in unmixed lists, the mixed list or within-subject design seems particularly appropriate for the study of strategies in discrimination problems. Indeed, as Kanak and Jones note, "practical discrimination problems often involve 'mixed-list' characteristics and certainly occur 'within-S's." (p.236).

\section{REFERENCES}

Bousfield, W. A., Whitmarsh, G. A., \& Danick, J. J. Partial response identities in verbal generalization. Psychological Reports, $1958,4,703-713$.

Cole, L \& Kanak, N. J. Transfer of implicit associative responses between free-recall learning and verbal discrimination learning tasks. Journal of Experimental Psychology, 1972, 95, 110-115.

Eberlein, E., \& Raskin, D. C. Intrapair and interpair associations in verbal discrimination learning. Psy chonomic Science, 1968. $11,145-146$.
Eckert, E., \& Kanak, N. J. Verbal discrimination learning: A review of the acquisition, retention, and transfer literature through 1972. Psy chological Bulletin, 1974, 81, 582.607.

Ekstrand, B. R. Wallace, W. P., \& Underwood, B. J. A frequency theory of verbal discrimination learning. Psycholugicial Review, $1966,73,566-578$.

Fulkerson, F. E., \& Kausler, D. H. Supplementary report: Effects of intrapair and interpair bidirectional associates on verbal discrimination learning. Journal of Verbal Learning and Verbal Behavior, 1969, 8, 307-310.

Kanak, N. J. The effects of rate of exposure upon simultalicuus intentional and incidental verbal discrimination learnin: Psychonomic Science, 1968, 12, 141-142.

Kanak, N. J., Cole, L. E., \& Eckert, E. Implicit associative responses in verbal discrimination acquisition. Journal of responses in verbal discrimination acquisition.

Kanak, N. J. \& Jones, K. N. Mixed-list manipulations of implicit associative responses in verbal discrimination leaming. Bulletin of the Psy chonomic Society, 1974, 3, 234-236.

Lovelace, E. A., \& Schulz, L. S. Intrapair associations in verbal discrimination learning. Psychonomic Science, 1971, 24, $157-158$.

McCarthy, S. V. Verbal discrimination leaming as a function of associative strength between noun pair members. Journal of Experimental Psy chology, 1973,97, 270-271

Palermo, D. S., \& Ullrich, J. R. Verbal discrinutiation learniub as a function of associative strength between wwid-pair members. Journal of Verbal Learning and Verbal Behavior, 1968, 7, 945-952. 